\title{
Navicular Stress Fractures: A Primer
}

\author{
Amol Saxena
}

\begin{abstract}
Navicular stress fractures (NSF) can be difficult injuries to diagnose and can end up career in athletes. The lack of blood supply to the navicular bone makes this injury difficult to heal and confoundingly gives minimal visible symptoms such as swelling and bruising.

Keywords: Foot and ankle, Navicular bone, Navicular stress fractures.

Journal of Foot and Ankle Surgery (Asia Pacific) (2020): 10.5005/jp-journals-10040-1127
\end{abstract}

Navicular stress fractures (NSF) can be difficult injuries to diagnose and can end up career in athletes. ${ }^{1,2}$ The lack of blood supply to the navicular bone makes this injury difficult to heal and confoundingly gives minimal visible symptoms such as swelling and bruising. ${ }^{3,4}$ Many patients, such as basketball players and dancers, complain of symptoms only when they are jumping or on their toes. This is why athletes relate they can jog but not sprint without pain and can therefore be difficult to diagnose early on..$^{5-10}$ In fact, one study showed on average it took almost 9 months to arrive at the correct diagnosis. ${ }^{6}$ Furthermore, despite magnetic resonance imaging (MRI) being a more common diagnostic test, it was helpful in correctly diagnosing NSF in $71 \%$ of patients, whereas computed tomography (CT) was $100 \%$ correlated with a correct diagnosis ${ }^{6}$ (Fig. 1).

According to Fitch et al., NSF was first recognized by Towne et al. in 1970. 4,11 Patients typically have pain in the midfoot, including the "N-spot" that is the high point of the navicular as well as vague anterior ankle and arch pain..$^{4,6,8}$ As noted previously, there is minimal swelling and bruising. This injury can occur after an ankle of midfoot sprain. ${ }^{6}$ Treatment from the 1970s time period until the end of the 1990s primarily involved nonsurgical care with a period of nonweight-bearing of 6-8 weeks in a below-knee cast. ${ }^{4,7,9,11}$ In 2000, Saxena et al. published their early results using a classification system using CT frontal plane images with $\leq 0.6$ $\mathrm{mm}$ slices. They described three stages of injury with progressive severity: type I, II, and III (Table 1). These authors added another
Department of Sports Medicine, Sutter Health-Palo Alto Medical Foundation, Palo Alto, California, USA

Corresponding Author: Amol Saxena, Department of Sports Medicine, Sutter Health-Palo Alto Medical Foundation, Palo Alto, California, USA, Phone: +1 650853 2943, e-mail: heysax@aol.com

How to cite this article: Saxena A. Navicular Stress Fractures: A Primer. J Foot Ankle Surg (Asia Pacific) 2020;7(2):62-63.

Source of support: Nil

Conflict of interest: None

less severe stage in 2011 termed type 0.5, which is essentially a stress reaction seen on MRI with no fracture identified via CT (12).

Table 1: Computed tomography Classification of navicular stress fractures (using $\leq 0.6 \mathrm{~mm}$ cuts in frontal plane)

Type $0.5=$ CT negative for fracture, MRI positive for signal change within body of navicular

Type I $=$ CT shows dorsal cortical fracture

Type II $=\mathrm{CT}$ shows fracture propagates into mid-portion of navicular

Type III = CT shows complete fracture through navicular

Based on Ref. 3 and 12
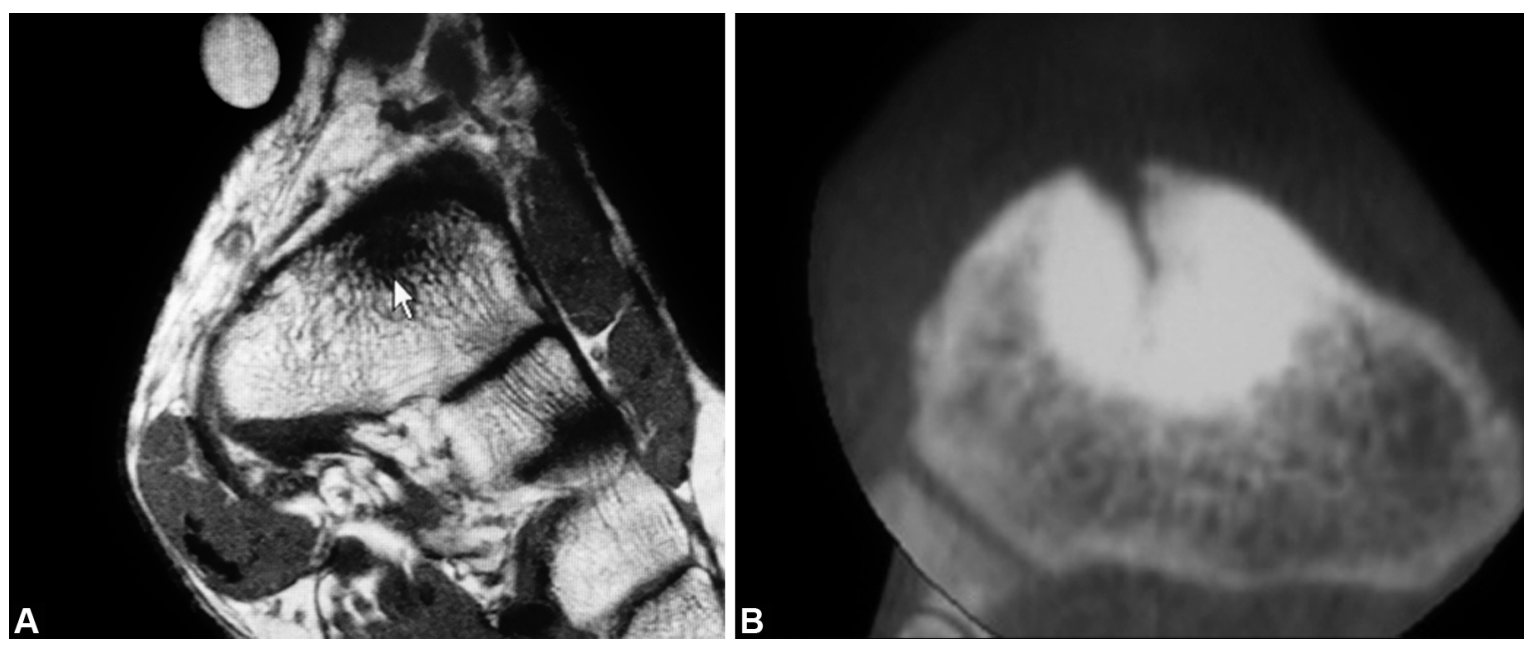

Figs 1 A and B: (A) Magnetic resonance imaging of navicular stress fracture failing to demonstrate fracture; (B) Computed tomography of same patient demonstrating type II navicular stress fractures

(-) The Author(s). 2020 Open Access This article is distributed under the terms of the Creative Commons Attribution 4.0 International License (https://creativecommons. org/licenses/by-nc/4.0/), which permits unrestricted use, distribution, and non-commercial reproduction in any medium, provided you give appropriate credit to the original author(s) and the source, provide a link to the Creative Commons license, and indicate if changes were made. The Creative Commons Public Domain Dedication waiver (http://creativecommons.org/publicdomain/zero/1.0/) applies to the data made available in this article, unless otherwise stated. 

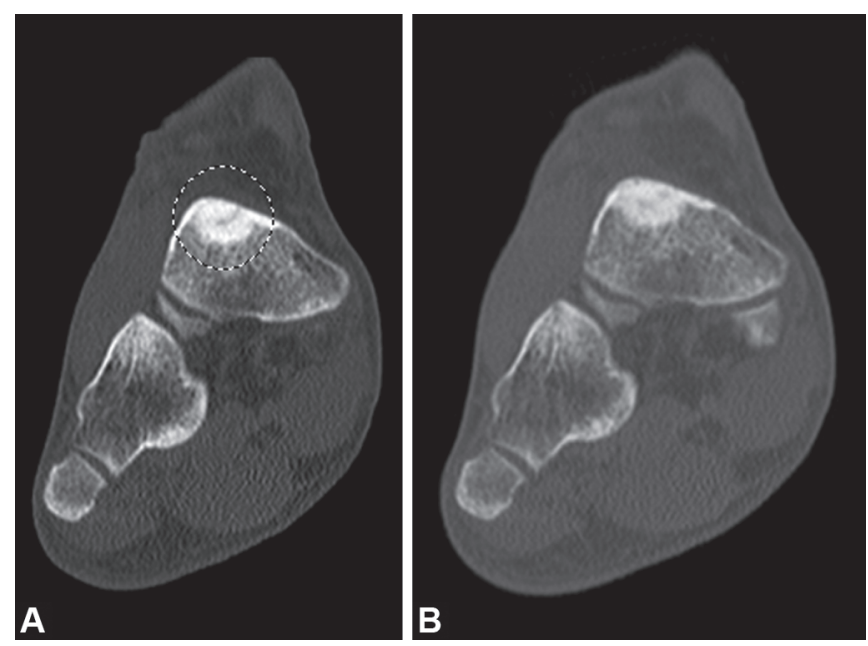

Figs 2A and B: (A) Computed tomography of a type I navicular stress fractures; (B) Same patient after two sessions of focused extracorporeal shock wave therapy and six weeks of nonweight-bearing in a boot

What Saxena et al. consistently showed in all their studies is, the more severe the injury (types II and III), the longer the healing time and the more likely surgery is recommended. ${ }^{3,6,8}$ In fact, in order to achieve a reasonable healing time (4 to 5 months), surgery is recommended for type II and III injuries. ${ }^{6,12}$ Studies have shown delayed treatment, even with surgery, is associated with less than ideal outcome, such as arthrosis, delayed union, refracture, and cessation of desired activity. ${ }^{1,2,5,6,10,12}$

Nonsurgical treatment is recommended for type 0.5 and I injuries. ${ }^{6,12}$ For type 0.5 , weight-bearing in a boot for 3-6 weeks appears acceptable, whereas for type I, in addition to immobilization a below-knee boot, a period of nonweight-bearing for 6 weeks, and 2-4 more weeks (until pain-free) is required. ${ }^{6}$ For athletes, a patient with a type I injury could also consider surgery, as some studies show faster healing with ORIF. ${ }^{3,6,8}$ Shockwave can be utilized for all navicular stress fractures as an adjunct; immobilization still should be performed ${ }^{13}$ (Fig. 2). For NSF types II and III, surgery is performed, typically with one or two $4.0 \mathrm{~mm}$ partially threaded screws, generally placed from lateral to medial but is certainly based on fracture pattern and can be modified. The incisional approach is typically lateral to the neurovascular bundle over the talonavicular joint. Autogenous bone graft can be utilized (usually from the lateral calcaneus) if there is sclerosis, cystic areas, avascular necrosis, and non-union. The same postoperative immobilization regimen what is used for type I injuries is used for types II and III. The average return to sports is 4 months, although full healing and activity can be longer. Refracture and persistent delayed union can be noted. Vitamin D status should be verified and supplemented as needed. Saxena et al.'s 2017 study in a large series of athletic patients had several interesting findings. Nonsurgical treatment in athletes under the age of 21 years appears more common with refracture. Screw removal was required in $11 \%$. Despite anatomic reduction, arthrosis of the adjacent joints can occur in $13 \% .{ }^{6}$ Instead of arthrodesis in an athlete, arthrodiastasis can be performed..$^{14}$ Treatment for avascular necrosis of an NSF can involve extracorporeal shock wave therapy (ESWT) and also a vascularized bone graft, although outcomes in athletes, particularly for the latter, is undocumented. ${ }^{15}$
Navicular stress fractures are significant injuries that involve significant downtime, possibly a loss of a season or worse. A provider should have a high index of suspicion for an athlete with persistent vague, atraumatic midfoot, or anterior ankle pain, particularly in the "N-spot". The consequence of missing this diagnosis and proper treatment is further downtime and poorer outcome, including re-fracture, arthrosis, and cessation of activity. Anecdotally, this author has seen associated medial ankle impingement with some cases of NSF. This association should be further studied. CT with $\leq 0.6-\mathrm{mm}$ cuts should be used to determine the type of injury. As of now, the proposed treatment recommendations of nonsurgical for types 0.5 and acute type I and ORIF for types II and III appear well supported in the literature. ESWT's role in accelerating healing appears favorable and deserves more research.

\section{References}

1. Anderson RB. Commentary on navicular stress fracture outcomes in athletes: analysis of 62 injuries. J Foot Ankle Surg 2017;56(5):949. DOI: 10.1053/j.jfas.2017.06.022.

2. Vopat B, Beaulieu-Jones BR, Waryasz G, et al. Epidemiology of navicular injury at the NFL combine and their impact on an athlete's prospective NFL career. Orthop J Sports Med 2017;5(8):2325967117723285 10.1177/2325967117723285.

3. Saxena A, Fullem B, Hannaford D. Results of treatment of 22 navicular stress fractures and a new proposed radiographic classification system. J Foot Ankle Surg 2000;39(2):96-103. DOI: 10.1016/s10672516(00)80033-2.

4. Fitch KD, Blackwell JB, Gilmour WN. Operation for non-union of stress fracture of the tarsal navicular. J Bone Joint Surg Br 1989;71(1):105-110. DOI: $10.1302 / 0301-620 X .71 B 1.2644288$.

5. Lee $S$, Anderson RB. Stress fractures of the tarsal navicular. Foot Ankle Clin 2004;9(1):85-104. DOI: 10.1016/S1083-7515(03)00151-7.

6. Saxena A, Behan SA, Valerio DL, et al. Navicular stress fracture outcomes in athletes: analysis of 62 injuries. J Foot Ankle Surg 2017;56(5):943-948. DOI: 10.1053/j.jfas.2017.06.009.

7. Fowler JR, Gaughan JP, Boden BP, et al. The non-surgical and surgical treatment of tarsal navicular stress fractures. Sports Med 2011;41(8):613-619. DOI: 10.2165/11590670-000000000-00000.

8. Saxena A, Fullem B. Navicular stress fractures: a prospective study on athletes. Foot Ankle Int 2006;27(11):917-921. DOI: 10.1177/107110070602701109.

9. Potter NJ, Brukner PD, Makdissi M, et al. Navicular stress fractures: outcomes of surgical and conservative management. Br J Sports Med 2006;40(8):692-695. DOI: 10.1136/bjsm.2005.022079.

10. Shakked RJ, Walters EE, O'Malley MJ. Tarsal navicular stress fractures. Curr Rev Musculoskelet Med 2017;10(1):122-130. DOI: 10.1007/s12178017-9392-9.

11. Towne LC, Blazina ME, Cozen LN. Fatigue fracture of the tarsal navicular J Bone Joint Surg Am 1970;52(2):376-378. DOI: 10.2106/00004623197052020-00023.

12. Saxena A, Fullem B. Comment on Torg et al, "management of tarsal navicular stress fractures: conservative versus surgical treatment". Am J Sports Med 2010;38(10):NP3-NP5. DOI: 10.1177/0363546510379341.

13. Leal C, D'Agostino C, Gomez Garcia S, et al. Current concepts of shockwave therapy in stress fractures. Int J Surg 2015;24(Pt B):195200. DOI: 10.1016/j.ijsu.2015.07.723.

14. Saxena A, Fullem BW. A unique procedure for treatment of osteochondral lesions of the tarsal navicular: three cases in athletes. J Foot Ankle Surg 2013;52(2):249-253. DOI: 10.1053/j.jfas.2012.10.019.

15. Fishman FG, Adams SB, Easley ME, et al. Vascularized pedicle bone grafting for nonunions of the tarsal navicular. Foot Ankle Int 2012;33(9):734-739. DOI: 10.3113/FAI.2012.0734. 